MIND THIEF 



\section{MIND THIEF}

THE STORY OF ALZHEIMER'S

\section{HAN YU}

Columbia University Press

New York 
Columbia University Press

Publishers Since 1893

New York Chichester, West Sussex

cup.columbia.edu

Copyright @ $2021 \mathrm{Han}$ Yu

All rights reserved

Library of Congress Cataloging-in-Publication Data

Names: Yu, Han, 1980- author.

Title: Mind thief : the story of Alzheimer's / Han Yu.

Description: New York : Columbia University Press, [2021] |

Includes bibliographical references and index.

Identifiers: LCCN 2020019015 (print) | LCCN 2020019016 (ebook) |

ISBN 9780231198707 (hardback) | ISBN 9780231552769 (ebook)

Subjects: LCSH: Alzheimer's disease.| Alzheimer's disease-History.

Classification: LCC RC523 .Y8 2021 (print) | LCC RC523 (ebook) |

DDC 616.8/311-dc23

LC record available at https://lccn.loc.gov/2020019015

LC ebook record available at https://lccn.loc.gov/2020019016

Columbia University Press books are printed on permanent and durable acid-free paper.

Printed in the United States of America

Cover design: Milenda Nan Ok Lee

Cover art: Spiderplay @ iStock 